\title{
PREMIUM CALCULATION WITHOUT ARBITRAGE? \\ A note on a contribution by $G$. Venter
}

\author{
By Peter Albrecht \\ Institute for Insurance Sciences, \\ University of Mannheim
}

\begin{abstract}
Stimulated by a recent contribution by G. Venter in this journal the adequateness of (re-)insurance premium calculation based on the hypothesis of arbitrage free (re-)insurance markets is questioned. It is argued that-in contrast to the theory of financial markets-it is not reasonable to demand that insurance markets are arbitrage free. In addition the adjusted distribution principles put forward by Venter are claimed to be invalid.
\end{abstract}

\section{KEYWORDS}

Premium calculation; no-arbitrage condition; financial insurance pricing.

\section{INTRODUCTION}

The application of results from modern financial theory to the problem of premium calculation of (re)-insurance companies (financial insurance pricing) has attracted much interest in recent years. For an extensive review of this field cf. Cummins (1990). It seems to be en vogue inside and outside actuarial circles to apply Capital Asset Pricing Models (CAPM), Option Princing Theory (OPT) and principles of Arbitrage Pricing (AP) to the problem of insurance premium calculation, often, at least in the author's opinion, rather uncritically and without consideration of the peculiarities of insurance markets and the specific nature of insurance business. As the application of CAPM and OPT has been criticized elsewhere, cf. Albrecht (1991a, 1991b), we will here concentrate on the question whether it is reasonable to assume that (re-) insurance markets satisfy the condition of no-arbitrage and to draw the corresponding implications from this hypothesis for insurance premium calculation, as done most recently by VENTER (1991). This starting point is not a new one, predecessors are e.g. BorCh (1982), Delbaen/HaEzendonck (1989), Kraus/Ross (1982) and SondermanN (1988). In addition, contributions, which apply OPT to insurance ratemaking rely heavily on the noarbitrage condition as well, cf. AlBRECHT (1991a).

The paper is organized as follows. First a general discussion on the adequateness of applying the no-arbitrage condition to (re-)insurance markets and insurance premium calculation is given. This discussion results in a negative 
conclusion. The paper then concludes with a discussion of some of the results of VENTER (1991).

\section{IS THE ARBITRAGE CONDITION A REASONABLE ASSUMPTION FOR INSURANCE MARKETS?}

The condition of the non-existence of (riskless) arbitrage possibilities in financial markets can be considered to be the central paradigm in modern financial theory for financial markets in price equilibrium under uncertainty, cf. e.g. VARIAN (1987). It is most successfully applied to a variety of problems, the most prominent may be option pricing. However, the relevant question for the present case is, whether it is a valid assumption that insurance markets are or should be arbitrage free (or close to being it) and whether implications from this assumption lead to reasonable conclusions. The main questions are:

(1) Under which conditions insurance markets will be arbitrage free? How can arbitrage opportunities be eliminated?

(2) What are the implications of arbitrage-free premium calculation principles?

Some answers to these questions are given in the following.

The condition of no-arbitrage can be formulated in very different ways in mathematical terms, cf. e.g. INGERSOLL (1987, Chapter 2). Intuitively in presence of arbitrage-freeness any two financial assets with completely identical characteristics (e.g. their return distribution in one-period models or their random cash flows in time continuous models) must have identical prices (market values) at any point of time. If this was not the case then by pure arbitrage operations riskless profits could be realized. In markets with complete information rational investors would exploit these possibilities until they are eliminated. Therefore it is reasonable to assume the non-existence of (riskless) arbitrage possibilities in capital market equilibrium. A direct consequence of this "law of one price" is that the price of a package of financial assets must be identical to the sum of the prices of the assets in the package, this is most clearly motivated in the paper of Cummins (1990, pp. 141-143). This gives the second central property of arbitrage free markets, they have to possess linear price structures. The price of a financial asset being a linear combination of other financial assets must be identical to the linear combination of the prices of these assets.

Summing up, the consequences of imposing the no-arbitrage condition to (re-)insurance markets are:

1) Identical risks will always be insured at the same price.

2) The relevant premium calculation principle applied by an (re-)insurance company must be an additive one, i.e.

$\pi\left(S_{1}+\ldots+S_{n}\right)=\pi\left(S_{1}\right)+\ldots+\pi\left(S_{n}\right)$,

where $\pi$ denotes the premium calculation principle applied and $S_{1}, \ldots, S_{n}$ are the accumulated claims of the risks insured. In contrast to risk theory, 
cf. e.g. Goovaer TS et al. (1984, p. 103), the additivity property is required for risks which are not stochastically independent, too.

Are these properties reasonable for insurance markets?

Ad 1):

It is not trivial, that identical risks are reasonably insured at the same price by every insurance company in the market. Insurance companies exhibit economies of scale with respect to risk taking, this means that companies with more security capital or larger collectives may--while operating at the same security level-insure risks at lower prices. BEARD et al. (1984, pp. 143-145) illustrate this property by proving, that the reserve funds of a company is dependent on the size of the company, e.g. a merged portfolio of independent risks requires less security capital than the separate portfolios together while still operating at the same security level.

Another straight forward example is the following. Let us fix the security level (only this assumption makes things reasonably comparable and assures that an identical product is sold which gives the same level of protection to the insured), neglect the security capital (its inclusion to the analysis is simple) and look at the idealistic situation that all risks are identically normal distributed (extension to the normal power case is straight-forward) and stochastically independent. We want to determine the adequate risk premium for an additional risk $X$ required by a company with a collective of $n$ risks $X_{1}, \ldots, X_{n}$ already insured at a collective risk premium $\pi\left(S_{n}\right)$ satisfying

$$
P\left(S_{n}>\pi\left(S_{n}\right)\right)=\varepsilon
$$

where $S_{n}=X_{1}+\ldots+X_{n}$ and $\varepsilon$ denotes the fixed security level of the company. Under the assumption of the normal distribution and the i.i.d. case $\pi\left(S_{n}\right)$ as defined in (1) is given by

$$
\pi\left(S_{n}\right)=n \mu+\sqrt{n} N_{\varepsilon} \sigma
$$

where $N_{\varepsilon}$ denotes the $(1-\varepsilon)$-quantile of the standard normal distribution and $\mu$ resp. $\sigma$ the mean resp. standard deviation of the normal distribution assumed.

Fixing the security level implies the condition

$$
P\left(S_{n}+X>\pi\left(S_{n}\right)+\pi(X)\right)=\varepsilon
$$

From (3) we obtain under the given assumptions

$$
\begin{aligned}
\pi\left(S_{n}\right)+\pi(X) & =E\left(S_{n}+X\right)+N_{\varepsilon} \sigma\left(S_{n}+X\right) \\
& =(n+1) \mu+N_{\varepsilon} \sqrt{n+1} \sigma
\end{aligned}
$$

With $\pi\left(S_{n}\right)$ given by (2) we finally obtain:

$$
\pi(X)=\mu+N_{\varepsilon}(\sqrt{n+1}-\sqrt{n}) \sigma
$$

As $f(n)=\sqrt{n+1}-\sqrt{n}$ is easily seen to be a strictly decreasing function in $n$ this simple example confirms our assertion, that a bigger insurance company 
(as measured by the number of insureds) can insure the same risk at a lower price, while still operating at the same level of security. As in reality insurance companies differ in size to a considerable extent this implies that in insurance markets the condition of no arbitrage would be a rather unnatural thing!

\section{Ad 2):}

The requirement of a strictly additive premium calculation principle again can be criticized from fundamental economic reasons. Using such a principle implies that the insureds take no advantage (in form of lower premiums) from the fundamental economies of scale effect with regard to risk taking as discussed above. The price of an individual risk would be the same whether there is no collective of identical risks already insured by the company or whether there is a collective of say 10.000 already insured identical risks present. The strict application of an additive premium calculation principle would severely reduce the economic advantages of buying insurance cover! Fixing again the security level, the premium $\pi$ of every risk $X$ would therefore be completely determined by

$$
P(X>\pi)=\varepsilon,
$$

which would result in a much too high premium for an individual risk in practice. Combining the property of strict additivity with the economical requirement of a sufficient security level of the company reveals that no arbitrage premiums are not reasonably at all for insurance markets. Beyond that, applying the no-arbitrage condition to insurance markets would not result in a competitive premium as opposed to the statement of Venter. In a competitive market insurance companies would consider the economies of scale effects discussed above when fixing their premiums, for instance a larger insurance company would give a larger premium reduction. As this effect is not considered by the no-arbitrage condition premiums would be generally too high (fixed at the level of an insurance company with a collective consisting of one insured only) and therefore completely non-competitive!

We close our discussion with examining the question under which conditions insurance markets could be arbitrage-free. VENTER (1991, p. 223) mentions the condition that transaction costs are ruled out or at least only have minimal effects. Further conditions are made explicit by DelBaEn/HaEZendoncK $(1989$, p. 269) "...in a liquid insurance market where products can be bought and sold very frequently and in different quantities, models of financial markets avoiding arbitrage opportunities may well be applied". This amounts to requiring continuous markets and perfect divisibility. Both conditions are far from the reality of insurance markets. Beyond that if in real markets one would try to come close to these conditions this would result in an explosion of transactions costs which in the case of (re-)insurance companies are those parts of the premium required for the cover of operating expenses, and this would be a contradiction to the assumption of negligible transaction costs. This in fact makes the existence of very liquid insurance markets with frequent trading of 
small risk portions virtually impossible. In addition the existence of IBNR (Insured But Not Reported) and IBNER (Insured But Not Enough Reserved) claims, which is a very important subject in property-liability insurance is another real-life reason why (re-)insurance markets can not have the structure assumed by financial theory. Clearly, the assumptions above are violated by empirical financial markets, too. But they are violated to a much lesser extent making the theoretical model a reasonable approximation to real markets. As shown above this is not the case for insurance markets.

In real financial markets arbitrage possibilities will exist, too, but it is argued that these possibilities will vanish or tend to zero, as certain investors (arbitrageurs) will realize the possibilities of a riskless profit. So, finally we have to analyse whether the arbitrage possibilities demonstrated above can be exploited in insurance markets.

Consider an (re-)insurance market with companies operating at an identical security level and with different sizes. As bigger companies will require lower premiums, a profit can only be made (neglecting transaction costs) by a transaction of the risk from a smaller to a bigger company. At the end of the process of exploiting all opportunities for arbitrage, all risks will be insured by the company being the biggest at the start of the process. The other companies will vanish from the market or they will again transfer all risks insured to 100 percent (pure fronting). As long as real markets are far from this situation, arbitrage opportunities will exist to a large extent (but cannot reasonably be exploited) and no-arbitrage premiums will tell nothing about real premiums required by real insurance companies.

Summing up the preceeding discussion we come to the following conclusion:

Statements on no-arbitrage premiums are completely non-informative for real insurance markets!

\section{SOME COMMENTS ON VENTER'S ADJUSTED DISTRIBUTION PRINCIPLES}

Complementary to the discussion above we in the following want to make some remarks on a class of "premium calculation principles" proposed by Venter on the basis of the no-arbitrage condition. VENTER (1991, p. 226) calls them "adjusted distribution principles".

The condition that arbitrage-free markets must have prices that follow a linear price functional (in the insurance case: an additive premium principle) only rules out some price functionals which are inadequate, namely non-linear ones. The adequate linear price functional still has to be identified and will depend on the specific economic theory and/or mathematical model used to formalize the empirical phenomenon. In case of the Capital Asset Pricing Model (CAPM) a specific Covariance Principle, in economic notation the security market line results. In case of option pricing theory several approaches exist for identifying the adequate option price, cf. the recent excellent survey (intended for actuaries) of SMITH (1991) on option pricing formulae. 
One generally applicable technique was discovered by Cox/Ross (1976). They showed that under certain conditions the adequate option price can be represented as an expected value, however, not with respect to the probability distribution of the financial asset underlying the option but with respect to a suitable transformation of this distribution, the so-called risk neutral distribution. The valuation formula can be formalized as

$$
\pi=\exp (-r T) E^{*}(X)
$$

where $E^{*}(\cdot)$ is the expectation operator associated with a probability measure $P^{*}$ which is equivalent (the two measures have the same null sets) to the original probability measure $P$. This was generalized by HARRISON/KREPS (1979) and HARRISON/PLISKA (1981), they analysed the valuation of contingent claims in terms of martingale theory and thus clarified the mathematical structure of the problem. They showed that a linear price system exists (i.e. the markets are arbitrage free) if and only if the discounted price process is a martingale under some probability measure $P^{*}$ equivalent to $P$. Prices of contingent claims are given by taking the mathematical expectation with respect to $P^{*}$. Implications of this are that the price process of the underlying security must be a semimartingale and the well developed theory dealing with change of measure for semimartingales, which has evolved from Girsanov's Theorem, cf. e.g. SмIтн (1991, p. 450) for Ito processes, is available.

VENTER (1991) attempts to capture the essence of these results when proposing his adjusted distribution premium principles as an adequate candidate for arbitrage-free insurance premiums. However his proposals lack an underlying theory and he gives only illustrative examples of transformed distributions (scale and power-scale transformations). VENTER (1991, p. 226) states: "Replacing the distribution by any other distribution will satisfy the additivity constraints". It would be my contention that this statement is too arbitrary to be the basis of what I would regard as a sound premium calculation principle. In my view the adequate transformed distribution, e.g. the risk neutral probability distribution as required in option pricing theory, has to be identified on the basis of a sound theory. So I would argue that, even when following the hypothesis of an arbitrage-free (re-)insurance market, Venter's results are invalid.

My point can be further supported as follows, by a slight variation of one of the paper's examples (this counter-example was communicated to the author by Thomas Mack). Venter e.g. proposes in case of a shifted Pareto severity distribution with distribution function $1-(1+\mathrm{x} / b)^{-2}$ to use a premium calculation principle which is based on the expected value of the shifted Pareto distribution but with parameter $b$ replaced by $1,1 b$. We use the same Pareto distribution for the loss variable $X$ but replace Venter's primary coverage up to the limit $10 b$, i.e. $g(X)=\min (X, 10 b)$, by a franchise cover, i.e.

$$
Y=g(X)=\left\{\begin{array}{lll}
X & \text { if } \quad X \leq c \\
0 & \text { if } \quad X>c
\end{array}\right.
$$


with $c<b$. Then we find $E(Y)=b(1+\mathrm{b} / c)^{-2}$ which is decreasing with increasing $b$ (as $c<b$ ). According to Venter the premium of this cover is obtained by replacing $b=E(X)$ by $1,1 b$. Because of the decreasing property of $E(Y)$ with respect to $b$ this implies

$$
\begin{aligned}
\pi(Y) & =1,1 b(1+1,1 b / c)^{-2} \\
& <b(1+b / c)^{-2}=E(Y),
\end{aligned}
$$

which contradicts to the most fundamental property of premium principles.

\section{SUMMARY}

There are two main lines of discussion in the present paper.

Section 2 questions the adequateness of incurance premium principles derived from the assumption of arbitrage-free (re-)insurance markets. The discussion is put forward in the spirit of a fundamental criticism. Not the formal derivation of results within a closed economical-mathematical model is questioned, but the adequateness of the model to explain something about real insurance markets or to obtain results useful for the management of an insurance company.

Section 3 argues that even when accepting the hypothesis of arbitrage-free (re-)insurance markets the adjusted distribution principles proposed by Venter are not derived on the basis of a sound theory.

\section{REFERENCES}

Albrecht, P. (1991a) Financial approach to actuarial risks? 2nd AFIR International Colloquium, Brighton, Vol. 4, 227-247.

ALBRECHT, P. (1991b) Kapitalmarkttheoretische Fundierung der Versicherung? Zeitschrift für die gesamte Versicherungswissenschaft 80, 499-530.

Beard, R.E., Pentikäinen, T. and Pesonen, E. (1984) Risk Theory. 3rd ed., Chapman and Hall.

BoRCH, K. (1982) Additive insurance premiums: A note. Journal of Finance 37, 1295-1298.

Cox, J.C. and Ross, S. (1976) The valuation of options for alternative stochastic processes. Journal of Financial Economics 3, 145-166.

Cummins, J.D. (1990) Asset pricing models and insurance ratemaking. ASTIN Bulletin 20, $125-166$.

Delbaen, F. and Haezendonck, J. (1989) A martingale approach to premium calculation principles in an arbitrage free market. Insurance: Mathematics and Economics 8, 269-277.

Goovaerts, M.J., De Vylder, F. and Haezendonck, J. (1984) Insurance Premiums, NorthHolland.

HARRISON, J. M. and KREPS, D. M. (1979) Martingale and arbitrage in multiperiod security markets. Journal of Economic Theory 20, 381-408.

Harrison, J. M. and Pliska, S. R. (1981) Martingales and stochastic integrals in the theory of continuous trading. Stochastic Processes and their Applications 11, 215-260.

Ingersoll, J.E. (1987) Theory of Financial Decision Making. Rowman \& Littlefield Publishers.

Kraus, A. and Ross, S.A. (1982) The determination of fair profits for the property-liability insurance firm. Journal of Finance 37, 1015-1028.

SMiтh, A. D. (1991) Option pricing formulae. 2nd AFIR International Colloquium, Brighton, Vol. 2, 415-452. 
Sondermann, D. (1988) Reinsurance in arbitrage free markets. Discussion Paper No. $13-82$. Department of Economics, University of Bonn.

VARIAN, H. R. (1987) The arbitrage principle in financial economics. Journal of Economic Perspectives $1,55-72$.

Venter, G.G. (1991) Premium calculation implications of reinsurance without arbitrage. ASTIN Bulletin 21, 223-230.

Peter Albrecht

Institute for Insurance Sciences, University of Mannheim, D-6800 Mannheim, Schloß. 